\title{
Optimizating Lighting Equipment of Substation Control Panel
}

\author{
Shen yongtao ${ }^{\mathrm{a}}$, Chen Liming ${ }^{\mathrm{b}}$, Pan Dong ${ }^{\mathrm{c}}$, Chen $\mathrm{Xin}^{\mathrm{d}}$, Zhao Jingru ${ }^{\mathrm{e}}$, Zhao \\ Qian', Wang Jiangang ${ }^{\mathrm{g}}$, Liu Hui ${ }^{\mathrm{h}}$, Huang Kai', Bao Wei', Guo Weik \\ State Grid Zhengzhou Power Supply Company , Zhengzhou of Henan province , 450000 \\ asyt19880927@163.com, bjianxiuclm@163.com, 'jianxiupandong@163.com

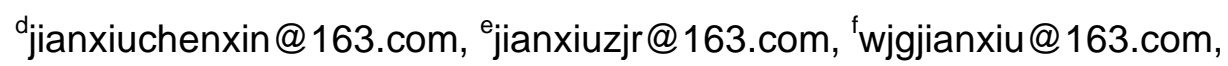 \\ gjianxiuzq@163.com, jianxiulh@163.com, jianxiuhk@163.com, \\ jjianxiubw@163.com, kjianxiugw@163.com
}

Keywords: LED; control panel; economic ; lighting; safe

Abstract. Combined with the actual situation, this paper presents a program of optimizing substation control panel lighting- using LED bulbs. Using LED lighting not only saves energy, but also save the maintenance or replacement of lighting equipment required manpower costs. At the same time, it is very friendly to the environment. The most important thing is that LED lightings provide a stable and reliable light. It can effectively avoid the wrong operation due to the blur sight, protect the safety of staff members, and protect the power grid safe and effective operation.

\section{Situation Analysis}

The control center room of substation completes the information collection, measurement, control, protection, measurement and monitoring, and other basic functions. It has an important role on the safe operation of the power system. At present, the control panel of the power supply company substation control room has been installed are mostly incandescent lamps and fluorescent lamps. Both lighting equipment have low illuminance, high energy consumption, short life expectancy, and high failure rate. According to the survey, Over 85 percent of substation control panel lighting have malfunctioned and failed to timely repair. Because there is no good lighting, it is necessary to assign specialized personnel to provide lighting when staff members work, resulting in a waste of human resources. It is shown in Fig.1.It brings inconvenience to daily inspection maintenance work of related staff members. What's more, it is harmful to the environment due to the high energy consumption of the lighting equipment, and staff members have not a clear line of sight due to the lack of lighting, it has serious implications for the personal safety and secure operation of the grid.
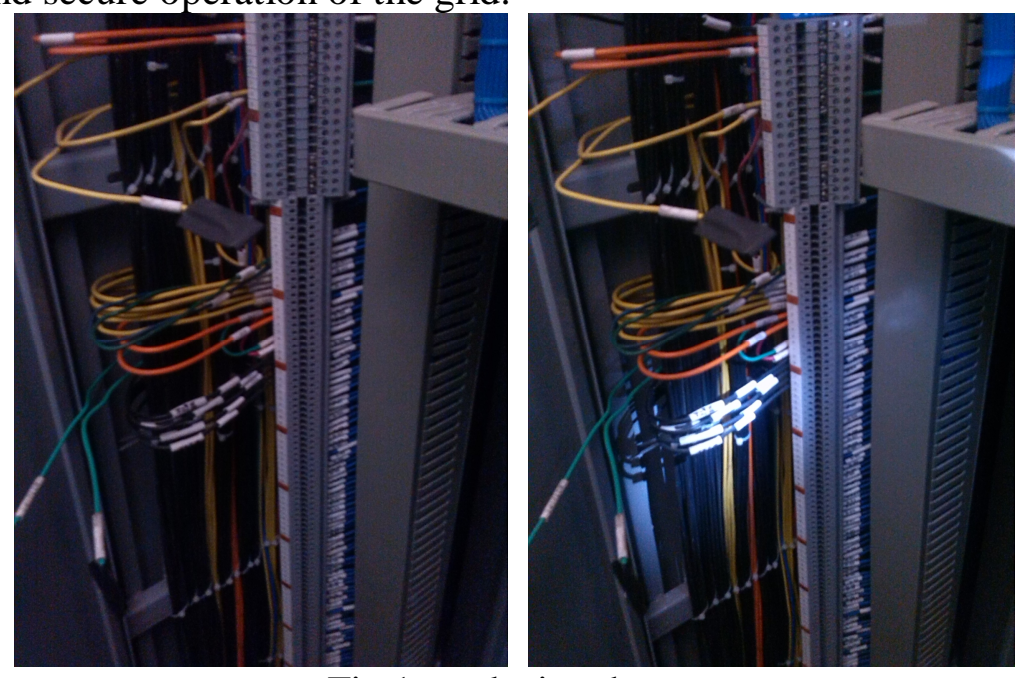

Fig.1 work site photos 


\section{Questions}

Combined with the actual situation, this paper presents a program of optimizing substation control panel lighting. It explores a lighting equipment that has a high illumination, low energy consumption, and long service life. This equipment can not only bring convenience to the work of the staff, protect environment, but also provide a reliable guarantee for the safety operation of electric power.

\section{Analyzing lighting equipments}

At present, the control panel of the power supply company substation control room has been installed are mostly incandescent lamps and fluorescent lamps.

The principle of incandescent lamps is that the filament is heated to an incandescent state and thermal radiation emits visible light. Only a very small part of the energy can be converted into useful energy in the conversion process. Incandescent lamp life is only about 1000 hours in general ${ }^{[1]}$. What's more, high temperature produced has a certain impact on the operation of the device, and accelerate the aging of equipment.The fluorescent lamp schematic is shown in Fig.2.

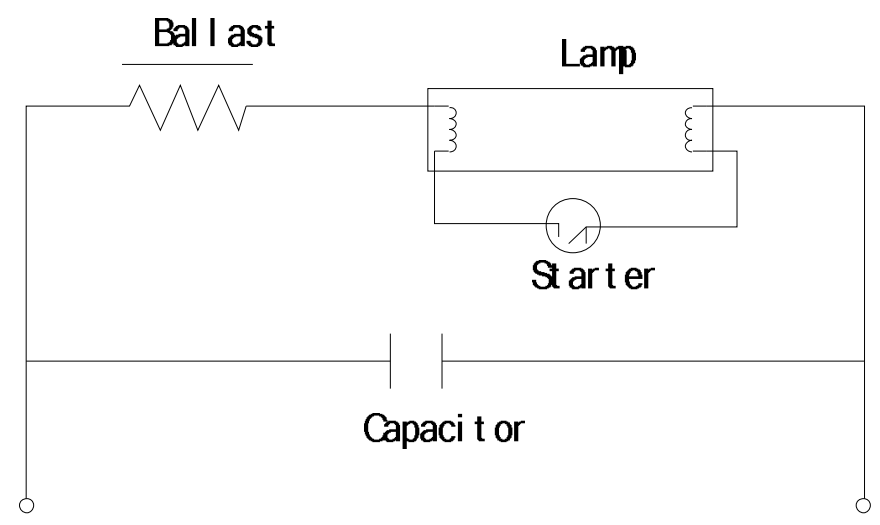

Fig.2 The fluorescent lamp schematic

When the switch is turned on, the power supply voltage is immediately applied to the poles of the starter by the ballast and lamp filament ${ }^{[2] .} 220 \mathrm{~V}$ voltage immediately ionizes the inert gas of the starter and produces glow discharge. The heat of the glow discharge makes the two metal plates heat and expand, and the poles contact. Current constitutes a path through the ballast, and the opening of the poles and the two ends of the filament. The filament is heated by the electric current and emits a large number of electrons. At this time, due to the closing of the poles, the voltage between the two poles is zero, the glow discharge disappears, the temperature of the tube is reduced, the double metal piece automatically reset and the poles disconnect. the circuit is cut off suddenly at the moment when poles is disconnected, and ballast produces great self-induced EMF overlapped with the supply voltage on both ends of the tube. When the filament is heated, a large number of electrons are emitted at the end of the tube and move from the high potential end to the low potential end with great speed. In the process of accelerating motion, electron collision tube argon molecules and make it ionize quickly.Argon ionization produces heat that causes the mercury to produce steam, and then the mercury vapor is ionized, and emits a strong ultraviolet light. Under the excitation of ultraviolet light, the fluorescent powder in the tube wall emits visible light near white.

At present in the field of lighting LED lamps are becoming more and more popular. LED (Emitting Diode Light), a light-emitting diode, is a solid-state semiconductor device that converts electrical energy into visible light, and it can be directly converted into light ${ }^{[3]}$. The heart of the LED is a semiconductor wafer, which is attached to a bracket at one end, and one end of the wafer is negative, and the other end is connected with the positive electrode of the power supply. Semiconductor chip is made up of two parts, a part of which is $\mathrm{P}$ type semiconductor, in which the hole occupies the dominant position, and the other end is $\mathrm{N}$ type semiconductor, which is mainly electronic. But when these two kinds of semiconductors are connected, they form a P-N knot. When current passes through the wire to 
the chip, the electrons are pushed into the $\mathrm{P}$ region, and the electrons in the $\mathrm{P}$ region are combined with the hole, and then emit energy in the form of a photon.

\section{Solution}

We can see through the introduction of a variety of lighting equipment, the work principle and the practical application experience: Incandescent lamp failure rate is high, the service life is short, the energy consumption is high, and high temperature produced has a certain impact on the operation of the device. It's unable to provide a stable and reliable lighting for the maintenance and maintenance personnel. Fluorescent lamp has mercury pollution in the production process and use of waste, and can not be a good response to the call of the state on the protection of the environment. What's more, The flicker of the fluorescent lamp is easy to make the human eye produce glare, which is not conducive to the maintenance work of the long time. LED lamp is small, low power consumption $(1 / 10,1 / 4$, fluorescent lamp) $^{[4]}$, non-toxic, environmental protection, light stability, low failure rate, long life (use up to 50000 hours or more). The price of LED lamp is higher than incandescent lamp and fluorescent lamp.

The economic benefits of incandescent lamp, fluorescent lamp and LED lamp are compared and analyzed in this paper. Access to relevant information, The lighting effect of 7WLED is equivalent to the effect of $100 \mathrm{~W}$ on the fluorescent lamp of incandescent lamp or $24 \mathrm{~W}^{[5]}$. Substation control panel replacement cycle is calculated for 5 years. Lighting time is 4 hours every day, then total 7300 years in five hours. 7WLED costs 40 RMB / only, and its life is 50000 hours, so in a circle there it needs 1 LED light bulbs; incandescent lamp costs 2 RMB / only, and its life is 1000 hours, so in a circle it needs 8 incandescent lamp light bulbs; fluorescent lamp costs $20 \mathrm{RMB} /$ only, and its life is 6000 hours, so in a circle it needs 2 fluorescent lamp light bulbs. LED lamp and fluorescent lamp, incandescent lamp use cost comparison is shown in Tab.1 (electric charge by 0.6 RMB / kWh).

Tab.1 the economic benefits of incandescent lamp, fluorescent lamp and LED lamp

\begin{tabular}{|c|c|c|c|}
\hline Cost types & 7W LED & 100W Incandescent lamp & Fluorescent \\
\hline $\begin{array}{c}\text { electricity } \\
\text { charge[RMB] }\end{array}$ & $\begin{array}{l}0.007 \mathrm{~kW} \times 7300 \mathrm{~h} \times \\
0.6 \mathrm{RMB} / \mathrm{kWh}=30.66\end{array}$ & $\begin{aligned} 0.1 \mathrm{~kW} & \times 7300 \mathrm{~h} \times 0.6 \\
\text { yuan } / \mathrm{kWh} & =438\end{aligned}$ & $\begin{array}{l}0.024 \mathrm{~kW} \times 7300 \mathrm{~h} \times 0.6 \\
\text { yuan } / \mathrm{kWh}=105.12\end{array}$ \\
\hline $\begin{array}{c}\text { Lamp } \\
\operatorname{cost}[\mathrm{RMB}]\end{array}$ & $1 \times 40 \mathrm{RMB}=40$ & $8 \times 2 \mathrm{RMB}=16$ & $2 \times 20 \mathrm{RMB}=40$ \\
\hline total[RMB & 70.66 & 454 & 145.12 \\
\hline
\end{tabular}

From the above table, we can see that the use of LED light bulb costs 70.66 RMB in a replacement cycle, Incandescent lamp will cost $454 \mathrm{RMB}$ (not including labor cost for maintenance and replacement of light bulbs), and Fluorescent lamps need to cost 145.12 yuan (not including labor cost for maintenance and replacement of light bulbs). According to the status quo of Zhengzhou power supply company, It needs to install or replace the lighting control panel about 1000.Then in terms of economic efficiency, LED lights save $1000 \times(454-70.66) \approx 380000$ RMB than incandescent, save $1000 \times($ $145.12-70.66$ ) $\approx 75000$ RMB than Fluorescent lamps.

At the same time, the energy consumption is reduced, and it is very friendly to the environment. Using the LED is very consistent with initiatives to protect the environment.

Considering the advantages of LED lights of Small size, low power consumption, non-toxic and environmentally friendly, light stability, low failure rate, long life, we suggest that the LED light bulb is used as the lighting equipment of the substation control panel in this paper. 


\section{Conclusion}

From the above analysis we know that using LED lighting not only saves energy, but also save the maintenance or replacement of lighting equipment required manpower costs. At the same time, it is very friendly to the environment. The most important thing is that LED lightings provide a stable and reliable light. It can effectively avoid the wrong operation due to the blur sight, protect the safety of staff members, and protect the power grid safe and effective operation.

\section{References}

[1] Peng Jun.Basic and application of optoelectronic devices.Science Press.2009,6

[2] Information on http://wenku.baidu.com/view/fe52a765f5335a8102d22085.html.

[3] Wang Shengxue, Wu Guangning, Jiang wei, Bian Shanshan, Li Shenglin. LED principle and its application. Lamps and lighting. .2007,30 ( 4 ) : 32-35, in Chinese.

[4] Yu Lihua.Electrical lighting.Tongji University press.2011,5.

[5] Jakovenko J,Formanek J,Janicek V,et al.High power solid state retrofot lamp thermal characterization and modeling. Radio Enjineering,2012,21(1):225-230,in Chinese. 